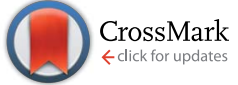

Cite this: Nanoscale, 2014, 6, 9681

\title{
Ultra-high-aspect-orthogonal and tunable three dimensional polymeric nanochannel stack array for BioMEMS applications $\uparrow$
}

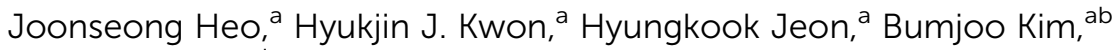 \\ Sung Jae Kim*d and Geunbae Lim*ae
}

\begin{abstract}
Nanofabrication technologies have been a strong advocator for new scientific fundamentals that have never been described by traditional theory, and have played a seed role in ground-breaking nanoengineering applications. In this study, we fabricated ultra-high-aspect $\left(\sim 10^{6}\right.$ with $\mathrm{O}(100) \mathrm{nm}$ nanochannel opening and $\mathrm{O}(100) \mathrm{mm}$ length) orthogonal nanochannel array using only polymeric materials. Vertically aligned nanochannel arrays in parallel can be stacked to form a dense nanostructure. Due to the flexibility and stretchability of the material, one can tune the size and shape of the nanochannel using elongation and even roll the stack array to form a radial-uniformly distributed nanochannel array. The roll can be cut at discretionary lengths for incorporation with a micro/ nanofluidic device. As examples, we demonstrated ion concentration polarization with the device for Ohmic-limiting/overlimiting current-voltage characteristics and preconcentrated charged species. The density of the nanochannel array was lower than conventional nanoporous membranes, such as anodic aluminum oxide membranes (AAO). However, accurate controllability over the nanochannel array dimensions enabled multiplexed one microstructure-on-one nanostructure interfacing for valuable biological/biomedical microelectromechanical system (BioMEMS) platforms, such as nanoelectroporation.
\end{abstract}

Received 19th January 2014

Accepted 9th June 2014

DOI: $10.1039 / \mathrm{c} 4 \mathrm{nr} 00350 \mathrm{k}$

www.rsc.org/nanoscale

\section{Introduction}

Recently, nanoscale fluidic channels have drawn significant attention in the fields of novel ion transportation, such as those associated with the nanofluidic rectification effect, ${ }^{\mathbf{1 , 2}}$ ion concentration polarization, ${ }^{3-5}$ and nanocapillarity. ${ }^{6}$ These new phenomena are the result of a critical surface effect, and can be applied to various engineering application fields, including entropic trapping for deoxyribonucleic acid (DNA), ${ }^{7}$ DNA manipulation in single nanochannels, ${ }^{\mathbf{8} 9}$ biosensors, ${ }^{\mathbf{1 0 , 1 1}}$ biofilters, ${ }^{\mathbf{1 2}}$ preconcentrations, ${ }^{\mathbf{1 3 , 1 4}}$ desalinations, ${ }^{15}$ and nanoelectroporations. ${ }^{16,17}$ To support their

\footnotetext{
${ }^{a}$ Department of Mechanical Engineering, Pohang University of Science and Technology, San 31, Pohang, Gyeongbuk, Republic of Korea.E-mail: limmems@postech.ac.kr; Tel: $+82-54-279-2186$

${ }^{b}$ Department of Electrical Engineering and Computer Science, Massachusetts Institute of Technology, 77 Massachusetts Avenue, Cambridge, MA 02139, USA

${ }^{c}$ Department of Electrical and Computer Engineering, Seoul National University, 1 Gwanak-ro, Gwanak-gu, Seoul, Republic of Korea. E-mail: gates@snu.ac.kr; Tel: +82-2-880-1665

${ }^{d}$ Inter-university Semiconductor Research Center, Seoul National University, Seoul 151-744, Republic of Korea

${ }^{e}$ Department of Integrative Bioscience and Biotechnology, Pohang University of Science and Technology, San 31, Pohang, Gyeongbuk, Republic of Korea

$\dagger$ Electronic supplementary information (ESI) available. See DOI: 10.1039/c4nr00350k
}

technological promise, numerous lithographic fabrication methods have been developed to build these systems, including atomic force microscopy (AFM) lithography, ${ }^{18}$ focused ion-beam milling, ${ }^{19}$ interference lithography, ${ }^{20}$ electron beam lithography, ${ }^{21}$ and nano-imprint lithography. ${ }^{22,23}$ The major advantages of these sophisticated nanofabrication technologies are their high resolution and reproducibility, leading to relevant technology for biological/biomedical microelectromechanical system (BioMEMS) applications. However, despite these advantages, high fabrication costs and complexity remain as issues limiting rapid prototyping and utilization in resource-limited settings. Additionally, while other fabrication technologies using anodic aluminum oxide $(\mathrm{AAO})^{12,24}$ and carbon nanotubes (CNTs) ${ }^{25,26}$ offer some advantages, such as relatively low fabrication costs and reliably large surface integration, these techniques require additional complex chemical treatments for biocompatibility.

In contrast, soft lithographic fabrication methods, which use soft materials such as polydimethylsiloxane (PDMS), have numerous advantages of simple, fast and cost effectiveness and utilizing standard microfabrication techniques. Whitesides et $a .^{27}$ introduced a soft lithographic method utilizing PDMS; PDMS has since become the representative material in microfluidic application fields, while it had a difficulty to apply nanofluidic application due to the flexibility. More recently, several groups have reported noteworthy fabrication processes 
using PDMS-based soft lithography methods. Takayama et al. ${ }^{28}$ developed a nanoscale-fracturing method of oxidized PDMS to fabricate nanofluidic systems that have nanochannel arrays with mechanically tunable widths. Similarly, shallow wrinkles on the boundary between stretched PDMS and a bare substrate have been used as nanofluidic channel arrays. ${ }^{29}$ Craighead et $a .^{30}$ demonstrated a technique for nanofluidic fabrication based on the controlled collapse of microstructures, i.e., the "roof-collapse" method. However, although these attempts used flexible, simple, low-cost nano-fabrication processes, their topological configurations were limited to two dimensions (low vertical aspect ratio). Three-dimensional (3-D) high-throughput applications, requiring high vertical aspect ratios, can rarely adopt these fabrication methods. In addition, applications that need specific properties of the nanochannel require other fabrication methods other than the aforementioned techniques. The properties include ultra-high aspect ratios, orthogonality, tunable shapes and sizes, large area integration, and low fabrication costs for the various nanofluidic BioMEMS applications of DNA manipulation, biomolecular preconcentration, nano-electroporation and nanofluidic biosensor technology. Ultra-high aspect ratio nanochannels have the advantage of enhancing the selectivity of DNA or biomolecules. $^{31,32}$ Straight, orthogonal nanochannels increase the transport rate of a species that passes through itself and provide appropriate model structures similar to mathematical (or numerical) models. Moreover, the nano-fabrication process of tunable shapes and sizes enables a fine-tune for a specific purpose such as circular-shaped nanochannels for bio-sensors ${ }^{\mathbf{1 0}}$ or vein mimicking, or triangular-shaped nanochannels for entopic focusing of DNA stretching, ${ }^{33}$ etc. In this study, we demonstrate an ultra-high aspect, orthogonal, tunable nanochannel fabrication process using only the soft PDMS material. The technique is suitable for on-demand, large-area, 3-D integration for high-throughput applications. The fabricated nanochannel-array structure was mainly fabricated by bonding of micro-bump arrays patterned on a PDMS surface and the flat surface of another PDMS film. The shape/size of the nanochannel was controlled through stretching and release of the PDMS during the fabrication process. Our nanochannel array structure offers superior advantages compared with those produced with conventional hard/soft lithographic methods. Table 1 includes an evaluation of the bottom-up fabrication strategy for $\mathrm{CNTs}^{25}$ and nano-material integration methods ${ }^{34,35}$ using Nafion and CNTs, ${ }^{36,37}$ as compared with the proposed method.

\section{Results and Discussion}

\section{Fabrication processes}

Fabrication method for polymeric nanochannel stack arrays (PNSAs). Fig. 1(a) and (b) show diagrams of the fabricationprocess flow. All molds for each PDMS-based film layer were fabricated using traditional photolithography: a silicon wafer was used as the substrate and negative photoresist, and SU8 (Microchem, Inc.) was used as the mold. ${ }^{27}$ The detailed fabrication processes for PNSA are described below.

Step 1(i): First, using conventional photolithography, we produced micro-sized, straight-lined SU8 molds (width: $20 \mu \mathrm{m}$; thickness: $2 \mu \mathrm{m}$; length: $6 \mathrm{~cm}$; pitch size: $20 \mu \mathrm{m}$ ); note that these dimensions could be varied, depending on the specific application. Bump arrays were patterned onto a silicon wafer. The thickness of the line pattern played a deterministic role in the nanochannel size.

Step 1(ii): A Teflon $0.1 \%$ solution (Dupont, Inc.) was spincoated onto the SU8 mold at $4000 \mathrm{rpm}$ to protect the patterns. The sample was then heated at $100{ }^{\circ} \mathrm{C}$ for $10 \mathrm{~min}$ using a hotplate. After curing the Teflon coating on the hot plate, a ringshaped guide film (8 $\mathrm{cm}$ in diameter) was glued to the SU8 mold.

Table 1 Comparison of conventional nanochannel fabrication technologies and our polymeric nanochannel stack array (PNSA)

\begin{tabular}{|c|c|c|c|c|c|c|c|c|c|}
\hline & \multicolumn{3}{|l|}{ HARD material } & \multicolumn{5}{|c|}{ SOFT material } & \multirow[b]{3}{*}{ Ours } \\
\hline & \multicolumn{2}{|l|}{ Top down } & \multirow{2}{*}{$\begin{array}{l}\text { Bottom up } \\
\text { CNT }^{25,26}\end{array}$} & \multicolumn{3}{|l|}{ PDMS } & \multicolumn{2}{|c|}{$\begin{array}{l}\text { Nanomaterial } \\
\text { integration }\end{array}$} & \\
\hline & Lithography $^{18-23}$ & $\begin{array}{l}\text { Porous alumina } \\
(\mathrm{AAO})^{12,24}\end{array}$ & & $\begin{array}{l}\text { Roof } \\
\text { collapsed }^{30}\end{array}$ & Cracking $^{28}$ & Wrinkling $^{29}$ & Nafion $^{34,35}$ & $\mathrm{CNT}^{36,37}$ & \\
\hline Density $\left(\# / \mathrm{mm}^{2}\right)$ &,$+++<\mathrm{O}\left(10^{9}\right)$ &,$+++<\mathrm{O}\left(10^{8}\right)$ &,$+++<\mathrm{O}\left(10^{11}\right)$ & + & + & + & +++ & +++ &,$+<\mathrm{O}\left(10^{4}\right)$ \\
\hline Shape/size control & ++++ & N/A & N/A & ++ & ++ & ++ & N/A & N/A & +++ \\
\hline $\begin{array}{l}\text { Vertical aspect } \\
\text { ratio }\end{array}$ &,$++<\mathrm{O}\left(10^{3}\right)$ &,$+++<\mathrm{O}\left(10^{3}\right) / \mathrm{h}$ &,$++++<\mathrm{O}\left(10^{8}\right)$ & + & + & + & + & + &,$++++<\mathrm{O}\left(10^{6}\right)$ \\
\hline 3D integration & +++ & +++ & + & + & + & + & ++ & + & +++ \\
\hline $\begin{array}{l}\text { Large area } \\
\text { integration }\end{array}$ & ++ & ++++ & +++ & + & + & + & +++ & +++ & ++++ \\
\hline Orthogonality & +++ & ++ & N/A & +++ & + & + & $\mathrm{N} / \mathrm{A}$ & $\mathrm{N} / \mathrm{A}$ & +++ \\
\hline Uniformity & +++ & ++ & + & ++ & ++ & ++ & + & + & ++ \\
\hline Fabrication time & + & + & + & +++ & +++ & +++ & +++ & ++ & +++ \\
\hline Flexibility & N/A & N/A & N/A & +++ & +++ & +++ & +++ & +++ & +++ \\
\hline Fabrication cost & + & ++ & + & +++ & +++ & +++ & ++ & ++ & +++ \\
\hline Biocompatibility & + & N/A & N/A & +++ & +++ & +++ & +++ & N/A & +++ \\
\hline
\end{tabular}


A Step 1 The fabrication of single PDMS fim i)

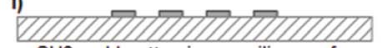
ii) SU

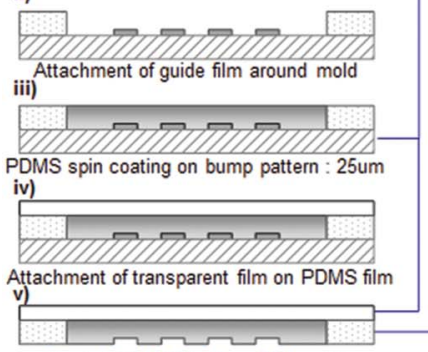
Peeled PDMS film from SU8 mold

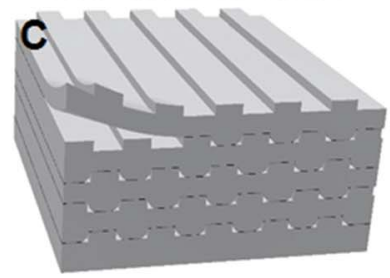

E
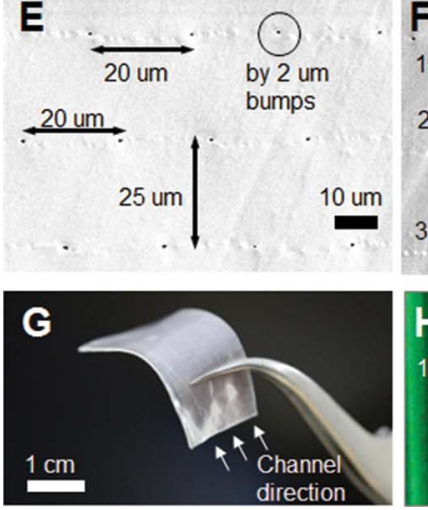

B

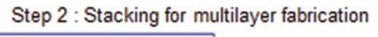
i)

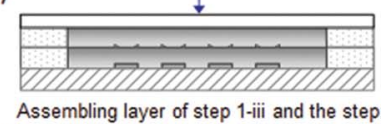
ii) $1-v$

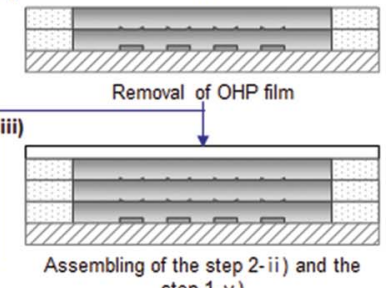
step 1-v)
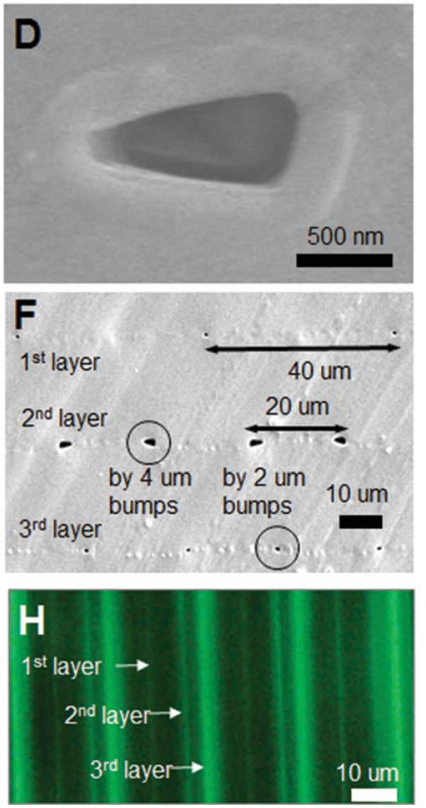

Fig. 1 Fabrication method and the resulting polymeric nanochannel stack array (PNSA). (a) Fabrication process flow of the PDMS film with bump patterns, (b) assembling process flow of the stacking method, and (c) schematic diagram of the PNSA. Each layer contained numerous nanochannels of constant pitch at each layer. (d) Scanning electron microscopy (SEM) image of individual nanochannel openings created by PDMS-to-PDMS bonding (bump height $=2 \mu \mathrm{m}$ ). (e) SEM image of the PNSA structure, including nanochannels having a constant pitch of $20 \mu \mathrm{m}$. Each layer had a thickness of $25 \mu \mathrm{m}$, created by spin-coating the PDMS mixture at a speed of $4000 \mathrm{rpm}$. (f) SEM image of PNSAs of various sizes and pitches at each inner layer. The first layer had a pitch of $40 \mu \mathrm{m}$; the second layer had a larger nanochannel than the third layer. (g) Image of the PNSA created by the aforementioned stacking technique. This structure has flexibility, an ultra-high aspect ratio, and orthogonality. (h) Fluorescent image inside the nanochannels of each inner layer.

Step 1(iii): A curing agent and PDMS solution (Sylgard 184 Silicone Elastomer Kit, Dow Corning) were mixed using a weight ratio of $1: 10$. The PDMS mixture was degassed at 40-50 mTorr in a desiccator for $1 \mathrm{~h}$ to guarantee complete mixing and to remove air bubbles from the mixture. The PDMS mixture was poured onto the SU8 mold master treated with Teflon coating, and was spin-coated at a speed of $4000 \mathrm{rpm}$ for $30 \mathrm{~s}$ to obtain a $25 \mu \mathrm{m}$ thick film. The sample was then heated on a $100{ }^{\circ} \mathrm{C}$

hotplate for $1 \mathrm{~h}$ to cure the PDMS film. The guide film in Step 1(ii) was a supporting layer for this thin PDMS-film coating.

Step 1(iv): A transparent plastic film (3M OHP film) was coated on the cured PDMS film; the sample was then heated on a $100{ }^{\circ} \mathrm{C}$ hotplate for $10 \mathrm{~min}$ to increase the adhesion between the OHP and PDMS films. The OHP film, in this case, played the role of a mechanical supporting layer, so that the PDMS films could be more easily peeled from the mold. Additionally, the OHP film provided a protective layer against contamination.

Step 1(v): The OHP-PDMS film was carefully peeled from the SU8 mold.

Step 2(i): The upper side of the PDMS film from Step 1(iii) and the bottom side of the OHP-PDMS film from Step 1(v) were treated with $\mathrm{O}_{2}$ plasma for permanent bonding (Cute-MP, Femto Science, Korea). The bonded layers were heated on a $100{ }^{\circ} \mathrm{C}$ hotplate for $1 \mathrm{~h}$. The high aspect ratio and orthogonal nanochannel arrays along the straight bump pattern were formed at the interface of these two PDMS layers. A typical nanochannel opening was on the order of $100 \mathrm{~nm}(\mathrm{O}(100) \mathrm{nm})$, and the length of the line pattern was typically $\mathrm{O}(100) \mathrm{mm}$; this produced nanochannels having an aspect ratio of $\sim 10^{6}$.

Step 2(ii): The OHP film was removed for further stacking.

Step 2(iii): A multilayered stack was fabricated by repeating this process. Each stack consisted of a nanochannel array.

Fig. 1(c) and (d) show diagrams of the PNSA layers and the opening of an individual nanochannel, respectively. The dimensions of the pitch between the nanochannels and the diameter of the nanochannel itself could be controlled by the bump patterns, as shown by comparing Fig. 1(e) and (f), leading to a controllable density of nanochannel arrays. Thus, using this technique, we were able to fabricate various sized nanochannels for each of the inner layers. Fig. 1(g) shows the fabricated PNSA nanochannel-array distribution, having an ultra-high aspect ratio, flexibility, and orthogonality. Stacking was confirmed by fluorescence measurements, by placing a dye (fluorescent sodium salt, Sigma Aldrich) inside the nanochannels (Fig. 1(h)). Because the fluorescence images were focused on the first layer, the other layers were out of the focal plane and therefore blurred. The channel structures were kept without disconnection in the middle of the high aspect ratio PNSA (see the ESI $\dagger$ ).

Large-area integration by PNSA rolling. The aforementioned stacking technique is inefficient due to the number of manual stacking cycles involved. We resolved this issue by rolling the PNSA array distribution for large-area integration. A special fabrication technique was required to roll a PNSA. A plastic bar was introduced for support. Before the PNSA was rolled, the first layer was coated with a PDMS layer made of a $1: 75$ weight ratio of the curing agent and the PDMS pre-polymer. As a result, the surface of the PNSA became sticky and soft to help the bonding since PNSA was relatively thick to have elasticity. The fabrication process of the 1 : 75 PDMS film was as follows. The PDMS mixture was poured onto a flat silicon wafer, treated with Teflon coating, and then spin-coated at a speed of $1000 \mathrm{rpm}$ for $50 \mathrm{~s}$ to obtain a $100 \mu \mathrm{m}$ thick film. The sample was then heated on a hotplate at $100{ }^{\circ} \mathrm{C}$ for $1 \mathrm{~h}$ to cure the PDMS film. Similar to the stack fabrication method, the PDMS film was peeled off using 
the guide film. One side of the sticky PDMS film layer (1: 75 PDMS film) and one side of the prepared PNSA film (length: 6 $\mathrm{cm}$; width: $1.5 \mathrm{~cm}$ ) were bonded after an air plasma treatment. Eventually, a double layer consisting of the sticky PDMS film on the PNSA film was obtained, as shown in the inset of Fig. 2(a). After plasma treatment of the sticky PDMS film, we rolled the PNSA film from its edge using a plastic bar. At this time, a small amount of uncured PDMS solution was glued for preventing a minute space which was unavoidably formed at the center of the roll due to the thickness of the PNSA film. One can roll the PNSA as much as one desires so that the diameter of the PNSA cylinder could be controlled and one can arbitrary cut the PNSA cylinder on demand as shown in Fig. 2(b) for large area integration. Fig. 2(c) and the magnified view shown in Fig. 2(d) confirm the radially and uniformly formed PNSA structure obtained using a PDMS film with a 1 : 75 weight ratio. Therefore, this technique potentially offers great versatility to be applied in the fields of DNA manipulation, bio-filter, bio/environmental-sensor, etc.

Tunable size and shape of a PNSA. Depending on the application, the specific configuration of the nanochannel array can be designed for particular applications, such as circular shapes for vein mimicking and bio-sensors, triangular shapes for entropic focusing of DNA, and size variations for bio/environmental filters. The present method can provide the tunable sizes and shapes utilizing the flexibility (or stretchability) of the PDMS material. We developed a controllable technique to elongate PDMS films during the bonding process. Because PDMS has high elasticity, the space created under a tensile force should shrink its volume after removing the force. Thus, we used this concept to demonstrate size/shape variations of nanochannel arrays as a function of the elongation rate (Fig. 3(a)). PDMS pieces, $2 \mathrm{~mm}$ in thickness, were prepared with a bump pattern of 2 or $4 \mu \mathrm{m}$ in height. Flat $25 \mu \mathrm{m}$-thick PDMS

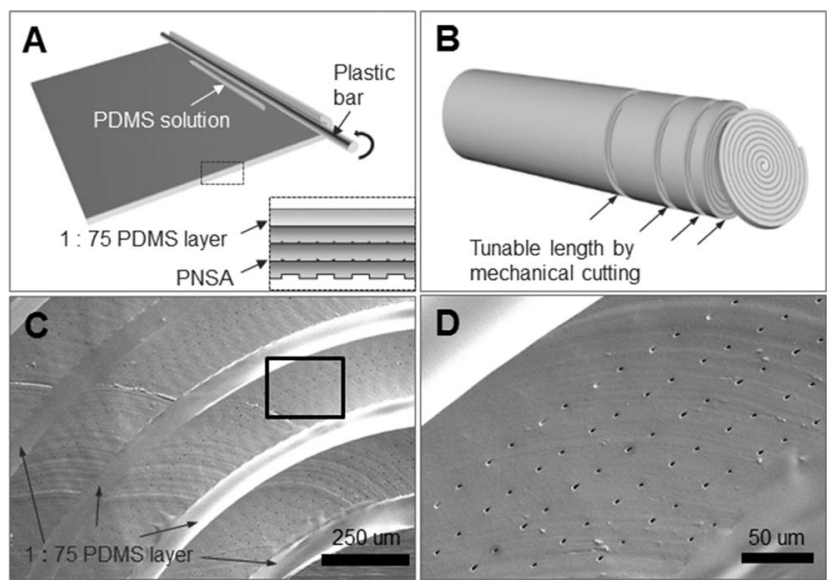

Fig. 2 Large-area integration by PNSA rolling. (a) Schematic illustration of large-area integration using a sticky (1:75) PDMS layer. Magnified inset image showing a cross-sectional view before rolling. (b) Schematic illustration of the rolled PNSA structure; this structure can be mechanically cut on demand. (c) SEM image of the radially formed PNSA and PDMS films of $1: 75$ weight ratio and (d) magnified view of (c).
A
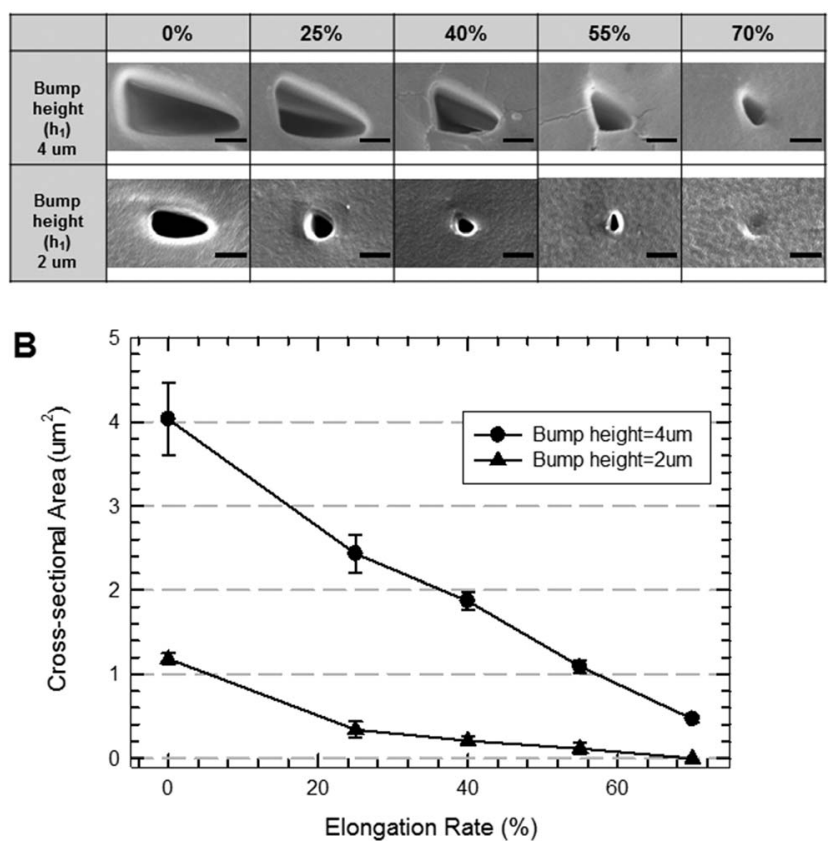

Fig. 3 Tunable size and shape of PNSA. (a) SEM image of the size/ shape variation of nanochannels as a function of the elongation rate (scale bar: $500 \mathrm{~nm}$ ). (b) Size characteristic curve of the cross-sectional area of the nanochannel according to the elongation rate.

films were also prepared. PDMS substrates, $2 \mu \mathrm{m}$ in thickness, were stretched to various elongations of $0,25,40,55$, and $70 \%$. The stretched $2 \mathrm{~mm}$ thick PDMS substrates and the flat $25 \mu \mathrm{m}$ thick PDMS films were bonded by plasma treatment. The elongation was removed after the bonding process.

Nanochannel arrays with a $4 \mu \mathrm{m}$ bump pattern were intentionally fabricated to clearly visualize the elongation effects. As the elongation increased, the shape of the nanochannel gradually changed. A circular nanochannel was observed at $40 \%$ elongation, and an elliptical shape was evident at 55\% elongation, for an initial triangular $2 \mu \mathrm{m}$ bump array state. Extreme elongation of over $70 \%$ eliminated the nanochannel formation with a $2 \mu \mathrm{m}$ bump array, as shown in the last image of the second row of Fig. 3(a). In addition to this shape variation, the size of the nanochannel was proportional to the inverse of the elongation rate, as shown in Fig. 3(b). The nanochannel size was analyzed as pixel numbers in the area using the ImageJ program. Therefore, the size and shape could be controlled by both the bump size and elongation rate. When the stacking of nanochannel arrays was combined with elongation, PNSA structures of different sizes, shapes, and densities for different layers could be achieved.

\section{Applications using PNSA}

Ion concentration polarization. Ion concentration polarization (ICP) is generated across ion-selective (or nanoporous) membranes under a direct-current (DC) bias due to the mismatch in ion transportation of the cations and anions. For negatively charged membranes, cations freely pass through the 
membrane; the transportation of anions is blocked. In this case, the resulting concentration profile in the vicinity of the membrane is depleted at the anode side (ion depletion zone) and enriched at the cathode side (ion enrichment zone). ${ }^{3,4,38}$ ICP has not only significant scientific impacts on nanoscale electrokinetics such as $2^{\text {nd }}$ kind electroosmotic flows and instabilities $^{\mathbf{2 , 3 8 - 4 0}}$ but also important engineering applicability with the combination of a tangential flow across the ion depletion zone. We developed a power-efficient desalination/purification/separation method with pressure-driven tangential flow ${ }^{15,41,42}$ and a multiplexed nanofluidic ion/preconcentration device. ${ }^{\mathbf{1 4 , 3 5 , 4 3}}$ Here, we demonstrate the applicability of PNSA for ICP applications.

The microchip for testing ICP consisted of three PDMS layers, as shown in Fig. 4(a). The lower and upper layers each had a microchannel (length: $2 \mathrm{~cm}$; width: $100 \mu \mathrm{m}$; height: 15 $\mu \mathrm{m})$. These two microchannels were orthogonally aligned at their centers, and a PDMS middle layer was sandwiched in between them. The middle layer had PNSA at the junction point of the upper and lower microchannels, such that the PNSA connected the two microchannels. After cutting the PNSA piece (length: $1.6 \mathrm{~mm}$; width: $1.6 \mathrm{~mm}$; thickness: $400 \mu \mathrm{m}$; 64-layer
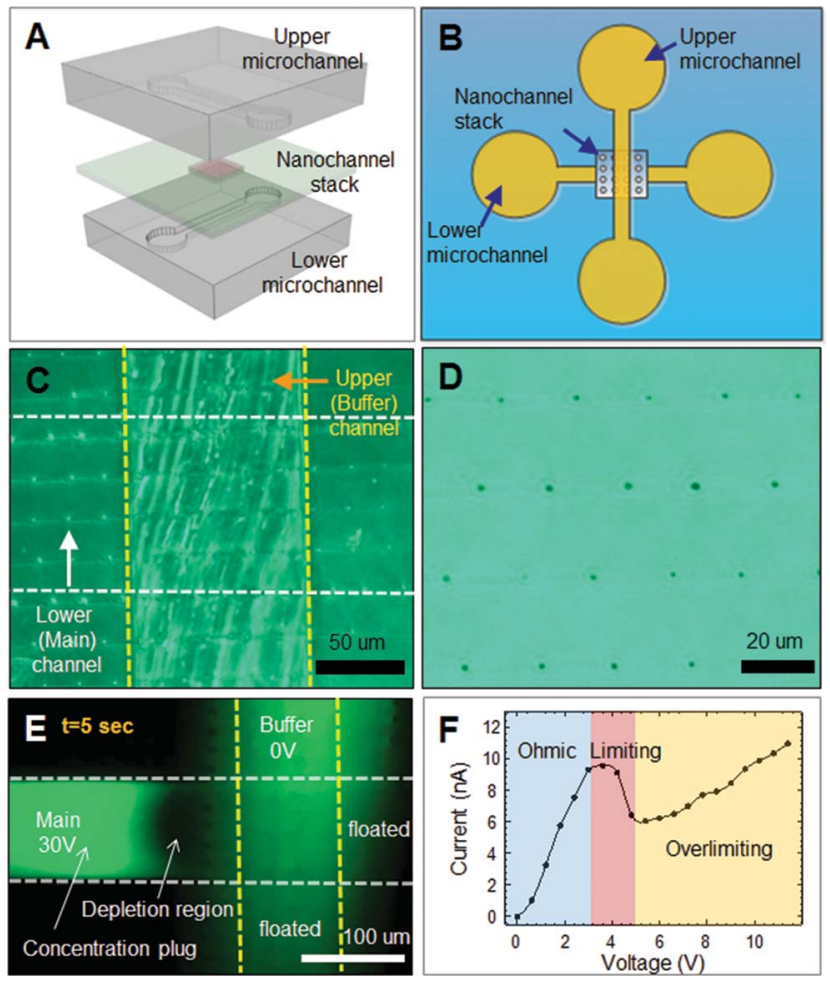

Fig. 4 lon concentration polarization (ICP) phenomenon using PNSA (a) Schematic illustration of the microchip used for testing ICP, which consisted of three PDMS layers. (b) Schematic illustration of the orthogonal alignment of each layer. (c) Microscopic image of the 3-D micro/nano-hybrid system. (d) Magnified view of PNSA at the junction of the upper and lower microchannels. (e) Fluorescent image of the development of the ion depletion zone by applying an external voltage of $30 \mathrm{~V}$ for $5 \mathrm{~s}$. (f) $I-V$ characteristics when applying an external electric field to the device; ohmic limiting and overlimiting regions appeared sequentially. nanochannel array), it was placed on 3M double-sided tape, which was attached to a glass slide. PDMS solution was placed around a piece of PNSA. We then flattened the PDMS solution via the capillary effect for $30 \mathrm{~min}$. The PDMS solution formed a PDMS film, which had the same height as the piece of PNSA. These three substrates were bonded as shown in Fig. 4(a); the alignment of each layer is shown in Fig. 4(b). Fig. 4(c) shows a microscopic image (IX71, Olympus, Japan) of the 3-D micro/ nano-hybrid system; the magnified view of the connected PNSA is shown in Fig. 4(d). In this set-up, 20 nanochannels (opening: $\sim 700 \mathrm{~nm}$; length: $400 \mu \mathrm{m}$ ) vertically connected the upper and lower microchannels. An electrical voltage was applied to the lower channel; the upper channel was electrically grounded for measurements of the current-voltage $(I-V)$ characteristics for the ICP phenomenon. The upper-layer microchannel acted as a buffer channel; the lower-layer microchannel was the main channel. For measuring the ionic conductance across the PNSA, both microchannels were filled with a buffer solution of $1 \mathrm{mM}$ $\mathrm{KCl}$; the buffer channel remained grounded over the course of the measurement.

When applying an external voltage of $30 \mathrm{~V}$ between the main and buffer channels, a negatively charged fluorescent dye (fluorescein sodium salt, $1 \mu \mathrm{g} \mathrm{mL} \mathrm{m}^{-1}$, Sigma Aldrich, $490 \mathrm{~nm}$ excitation and $520 \mathrm{~nm}$ emission) exerted a repulsive force from the nanojunction (Fig. 4(e)), creating an ion depletion zone. The development of the ion depletion zone proved that the permselectivity of the PNSA drives the ICP phenomena. Because the east and south reservoirs floated, the depletion boundary appeared only at the left side of the main channel, and the fluorescent intensity increased at the buffer channel by the formation of an ion enrichment zone, demonstrating the role of PNSA as a nanoporous membrane. Because ICP leads to a dynamic, nonlinear change in the electrical resistance, a limiting and overlimiting current region should appear next to the ohmic current range in the $I-V$ measurements. ${ }^{\mathbf{4} 44}$ An electrical voltage of $0-12 \mathrm{~V}$ was applied to the main channel using a Keithley $238 \mathrm{SMU}$; the voltage increased linearly at a rate of 600 $\mathrm{mV}$ per $10 \mathrm{~s}$ without any external pressure. Fig. 4(f) shows that a limiting zone appeared between 3 and $5 \mathrm{~V}$ by the depletion region formation; the overlimiting zone was initiated for voltages exceeding $5 \mathrm{~V}$. These outcomes indicate that the dominant permselectivity for ICP originated from the PNSA.

Multiplexed one microstructure-on-one nanostructure interfacing. While our PNSA structure provides orthogonal nanochannels with an ultra-high-aspect ratio, the spacing among nanochannels should be at least $\mathrm{O}(10) \mu \mathrm{m}$, i.e., a low nanochannel density, as shown in Table 1 . However, certain applications require sparse arrays of nanochannels, with constant spacing $(>10 \mu \mathrm{m})$ to interface a micron-size object with a single nanochannel; these applications include nanoelectroporation $^{\mathbf{1 7}}$ that enhances DNA and biomolecule electroporation efficiency through a cell using a nanochannel. The electric field can be focused to a nanosized spot of the cell surface, greatly improving the injection speed and accuracy. In such applications, the ability to interface a single cell with only a single nanochannel, without any disturbance from neighboring nanochannels, is critical. Additionally, a multiplexed 
scheme is highly desirable to achieve high throughput. PNSA provides the most appropriate structure for satisfying these issues. Compared with our PNSA technique, hard material processes (e.g., e-beam lithography and focused ion-beam milling) are costly, time-consuming and complex. While the nanoholes using the AAO nanofabrication method have a significantly higher density of nanochannels, the appropriate one microstructure-on-one nanostructure interfacing is not feasible due to the high density.

Fig. 5(a) shows the interfacing scheme using a support layer on top of the PNSA. The support layer was made of a $15 \mu \mathrm{m}$ thick SU8 film, and had a number of aligned $15 \mu \mathrm{m}$ diameter chambers. Each chamber was aligned with a single nanochannel of PNSA; in this way, a micro-sized particle would be in contact with a single nanochannel and could be trapped in the
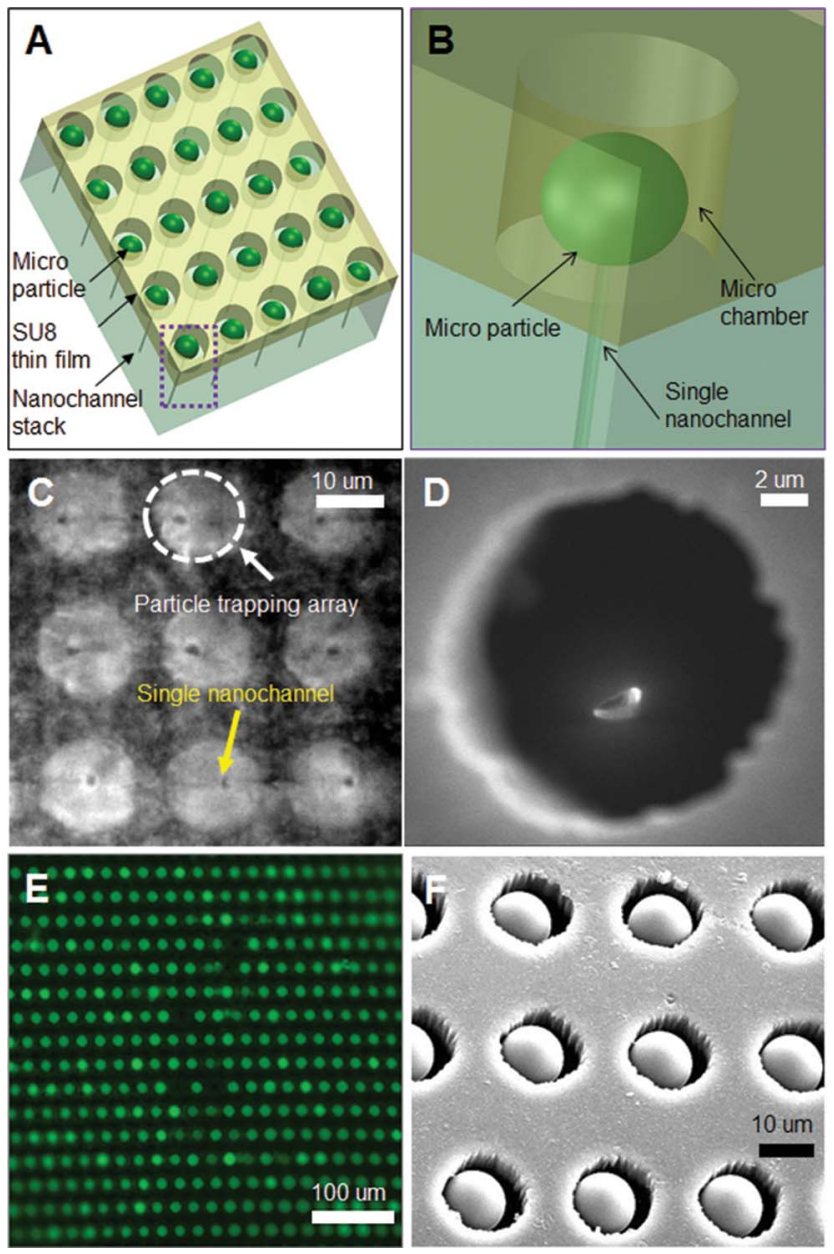

Fig. 5 Multiplexed one microstructure-on-one nanostructure interfacing using PNSA. (a) Schematic illustration of micro-particle/nanochannel interfacing using a support layer on top of PNSA. (b) Magnified schematic view of one chamber loaded with one microparticle. (c) Microscopic image of the positioning of a single nanochannel with the microchamber. Two images are overlaid: one shows the nanochannel and the other shows the microchamber. (d) SEM image of a single chamber aligned on top of a single nanochannel. (e) Fluorescent image of multiplexed one microstructure-on-one nanostructure interfacing of fluorescent particles and nanochannels. (f) SEM image of (e). chamber. A magnified view of one chamber loaded with one microparticle is shown in Fig. 5(b). The positioning of a single nanochannel with the micro-chamber was confirmed by the microscopic images shown in Fig. 5(c) and the scanning electron microscopy (SEM) image shown in Fig. 5(d). Fig. 5(c) shows two images (one of the nanochannel and the other of the microchamber) superimposed for a clear visualization. A droplet containing a number of $9.9 \mu \mathrm{m}$ diameter particles (fluorescent polystyrene particles similar in size to a cell, Thermo Scientific Corp.) was placed on top of the device; individual particles gradually sank and were collected in the microchamber. This one microstructure-on-one nanostructure interfacing was confirmed by the microscopic image shown in Fig. 5(e) and the SEM image shown in Fig. 5(f). Therefore, one can utilize PNSA, with a micro-chamber guide layer in the application, for multiplexed one microstructure-on-one nanostructure interfacing.

\section{Conclusion}

In this study, with minimal lithographic steps, we fabricated ultra-high-aspect ratio, orthogonal nanochannel 3-D arrays using only flexible polymeric materials. The vertical aspect ratio could be varied up to $\sim 10^{6}$, with $\mathrm{O}(100) \mathrm{nm}$ and $\mathrm{O}(100) \mathrm{mm}$ the opening and length, respectively. Compared with previously reported nanohole arrays, the nanochannels were straight, while the cross-sectional shape and size could be tuned using the elongation method. While the fabrication method of PNSA is similar to the roof collapse method, the stacking and rolling method is the major point that we are trying to convey. This method can extend the versatility of polymer based nanochannels, since one can easily obtain multiplexed nanostructures without sacrificing advantages such as ultra-highaspect-ratio, orthogonality, etc. The ability to roll and cut the stacked nanochannel arrays at one's own discretion provides a valuable experimental platform for biomolecular manipulation, bio/environmental-filters, and sensors. As examples, we demonstrated the applicability of these platforms to nanofluidic ICP operation and multiplexed one microstructure-onone nanostructure interfacing. Additionally, we believe that soft material processing (Table 1) could be extended to 3-D multiplexed schemes using our stacking and rolling method.

\section{Methods}

\section{Device fabrication}

We fabricated an SU8 (Microchem, Inc) mold with bump patterns on a silicon wafer using conventional photolithography. Each bump of various thicknesses was made from a diluted SU8 photoresist with a SU8 thinner (Microchem, Inc). The thicknesses of the bumps were confirmed by Alpha-Step surface profiler measurements. For measurement of the $I-V$ characteristics of ICP, the main and buffer microchannels had dimensions of $100 \mu \mathrm{m}$ (width) $\times 15 \mu \mathrm{m}$ (depth); the distance between the two channels was $400 \mu \mathrm{m}$ (the thickness of the middle layer, including the PNSA). We mechanically punched 
holes in the microchannel reservoirs before PDMS-to-PDMS bonding.

We fabricated a $15 \mu \mathrm{m}$ thick SU8 film as a support layer for the multiplexed one microstructure-on-one nanostructure interfacing test. The AZ9260 photoresist was spin-coated at a speed of $4000 \mathrm{rpm}$ for $40 \mathrm{~s}$ to obtain $5 \mu \mathrm{m}$ film thicknesses. A 15 $\mu \mathrm{m}$ thick SU8 film, including a number of aligned $15 \mu \mathrm{m}$ diameter chambers, was patterned on the AZ9260 film using conventional photolithography. To obtain only a SU8 film, the fabricated mold pattern was soaked in acetone. When soaked in acetone, a number of $15 \mu \mathrm{m}$ diameter chambers played the role as etch holes for etching of the AZ9260 film. After the SU8 film was rinsed in methanol, it was dried using isopropyl alcohol.

\section{Materials}

The main buffers of $\mathrm{KCl}$ (Sigma Aldrich) were prepared at $1 \mathrm{mM}$ concentrations. For visualizing flow patterns and the depletion zone, fluorescein sodium salt $\left(1 \mu \mathrm{g} \mathrm{mL}{ }^{-1}\right.$, Sigma Aldrich, 490 $\mathrm{nm}$ excitation and $520 \mathrm{~nm}$ emission) was added to the main buffers.

\section{Methods}

$I-V$ sweep plots through fabricated PNSAs were measured using a source measurement unit (Keithley 238); the voltage was applied over the range of $0-12 \mathrm{~V}$ at a rate of $0.6 \mathrm{~V}$ per $10 \mathrm{~s}$. For tracking the depletion zone and concentrated areas, we used an inverted fluorescent microscope (Olympus IX71) and a chargedcoupled device (CCD) camera (Olympus DP 72). Open-source software, ImageJ, was used for the image analysis. All measurements were repeated at least 10 times for confirmation of repeatability.

\section{Conflict of interest}

The authors declare no competing financial interest.

\section{Acknowledgements}

This work was supported by the National Research Foundation of Korea (NRF) grant funded by the Korea government (MEST) (Grant no. 2012R1A2A2A06047424). We gratefully appreciate Dr H. Lee's contribution for his valuable discussions on this work.

\section{References}

1 H. Daiguji, Chem. Soc. Rev., 2010, 39, 901-911.

2 B. Kim, J. Heo, H. J. Kwon, S. J. Cho, J. Han, S. J. Kim and G. Lim, ACS Nano, 2013, 7, 740-747.

3 R. F. Probstein, Physicochemical Hydrodynamics : An Introduction, Wiley-Interscience, 1994.

4 S. J. Kim, Y.-C. Wang, J. H. Lee, H. Jang and J. Han, Phys. Rev. Lett., 2007, 99, 044501.

5 T. A. Zangle, A. Mani and J. G. Santiago, Chem. Soc. Rev., 2010, 39, 1014-1035.

6 J. W. van Honschoten, N. Brunets and N. R. Tas, Chem. Soc. Rev., 2010, 39, 1096-1114.
7 J. Han and H. G. Craighead, Science, 2000, 288, 1026-1029.

8 S. L. Levy and H. G. Craighead, Chem. Soc. Rev., 2010, 39, 1133-1152.

9 F. Persson and J. O. Tegenfeldt, Chem. Soc. Rev., 2010, 39, 985-999.

10 A. de la Escosura-Muniz and A. Merkoci, Electrochem. Commun., 2010, 12, 859-863.

11 A. de la Escosura-Muniz and A. Merkoci, Small, 2011, 7, 675682.

12 S. Lee, M. Park, H.-S. Park, Y. Kim, S. Cho, J. H. Cho, J. Park and W. Hwang, Lab Chip, 2011, 11, 1049-1053.

13 Y.-C. Wang, A. L. Stevens and J. Han, Anal. Chem., 2005, 77, 4293-4299.

14 S. J. Kim, Y.-A. Song and J. Han, Chem. Soc. Rev., 2010, 39, 912-922.

15 S. J. Kim, S. H. Ko, K. H. Kang and J. Han, Nat. Nanotechnol., 2010, 5, 297-301.

16 S. Wang and L. J. Lee, Biomicrofluidics, 2013, 7, 011301.

17 P. E. Boukany, A. Morss, W.-C. Liao, B. Henslee, H. Jung, X. Zhang, B. Yu, X. Wang, Y. Wu, L. Li, K. Gao, X. Hu, X. Zhao, O. Hemminger, W. Lu, G. P. Lafyatis and L. J. Lee, Nat. Nanotechnol., 2011, 6, 747-754.

18 L. Pellegrino, Y. Yanagisawa, M. Ishikawa, T. Matsumoto, H. Tanaka and T. Kawai, Adv. Mater., 2006, 18, 3099-3104.

19 H. Cao, Z. Yu, J. Wang, J. O. Tegenfeldt, R. H. Austin, E. Chen, W. Wu and S. Y. Chou, Appl. Phys. Lett., 2002, 81, 174-176.

20 M. J. O'Brien, P. Bisong, L. K. Ista, E. M. Rabinovich, A. L. Garcia, S. S. Sibbett, G. P. Lopez and S. R. J. Brueck, J. Vac. Sci. Technol., B: Microelectron. Nanometer Struct.Process., Meas., Phenom., 2003, 21, 2941-2945.

21 C. H. Reccius, S. M. Stavis, J. T. Mannion, L. P. Walker and H. G. Craighead, Biophys. J., 2008, 95, 273-286.

22 Q. Xia, K. J. Morton, R. H. Austin and S. Y. Chou, Nano Lett., 2008, 8, 3830-3833.

23 M. D. Austin, H. X. Ge, W. Wu, M. T. Li, Z. N. Yu, D. Wasserman, S. A. Lyon and S. Y. Chou, Appl. Phys. Lett., 2004, 84, 5299-5301.

24 W. Lee, R. Ji, U. Gosele and K. Nielsch, Nat. Mater., 2006, 5, 741-747.

25 J. K. Holt, H. G. Park, Y. Wang, M. Stadermann, A. B. Artyukhin, C. P. Grigoropoulos, A. Noy and O. Bakajin, Science, 2006, 312, 1034-1037.

26 G. Zhong, J. H. Warner, M. Fouquet, A. W. Robertson, B. Chen and J. Robertson, ACS Nano, 2012, 6, 2893-2903.

27 D. C. Duffy, J. C. McDonald, O. J. A. Schueller and G. M. Whitesides, Anal. Chem., 1998, 70, 4974-4984.

28 D. Huh, K. L. Mills, X. Zhu, M. A. Burns, M. D. Thouless and S. Takayama, Nat. Mater., 2007, 6, 424.

29 S. Chung, J. H. Lee, M.-W. Moon, J. Han and R. D. Kamm, Adv. Mater., 2008, 20, 3011-3016.

30 S.-M. Park, Y. S. Huh, H. G. Craighead and D. Erickson, Proc. Natl. Acad. Sci. U. S. A., 2009, 106, 15549-15554.

31 P. Mao and J. Han, Lab Chip, 2009, 9, 586-591.

32 J. Fu, R. B. Schoch, A. L. Stevens, S. R. Tannenbaum and J. Han, Nat. Nanotechnol., 2007, 2, 121-128. 
33 W. F. Reinhart, D. R. Tree and K. D. Dorfman, Biomicrofluidics, 2013, 7, 024102.

34 S. J. Kim and J. Han, Anal. Chem., 2008, 80, 3507-3511.

35 S. H. Ko, S. J. Kim, L. Cheow, L. D. Li, K. H. Kang and J. Han, Lab Chip, 2011, 11, 1351-1358.

36 C. Y. Lee, W. Choi, J.-H. Han and M. S. Strano, Science, 2010, 329, 1320-1324.

37 W. Choi, Z. W. Ulissi, S. F. E. Shimizu, D. O. Bellisario, M. D. Ellison and M. S. Strano, Nat. Commun., 2013, 4, 2397. 38 S. J. Kim, S. H. Ko, R. Kwak, J. D. Posner, K. H. Kang and J. Han, Nanoscale, 2012, 4, 7406-7410.
39 P. Kim, S. J. Kim, J. Han and K. Y. Suh, Nano Lett., 2010, 10, 16-23.

40 I. Cho, G. Y. Sung and S. J. Kim, Nanoscale, 2014, 6, 46204626.

41 R. Kwak, S. J. Kim and J. Han, Anal. Chem., 2011, 83, 73487355.

42 H. Jeon, H. Lee, K. H. Kang and G. Lim, Sci. Rep., 2013, 3, 3483.

43 S. H. Ko, Y.-A. Song, S. J. Kim, M. Kim, J. Han and K. H. Kang, Lab Chip, 2012, 12, 4472-4482.

44 I. Rubinstein and L. Shtilman, J. Chem. Soc., Faraday Trans. 2, 1979, 75, 231-246. 